\title{
Links between accelerated replicative cellular senescence and down-regulation of SPHK1 transcription
}

\author{
Min Kyung Kim ${ }^{1}$, Wooseong Lee ${ }^{1}$, Gang-Ho Yoon ${ }^{2}$, Eun-Ju Chang ${ }^{1,2}$, Sun-Cheol Choi ${ }^{1,2, *} \mathcal{E}^{*}$ Seong Who Kim ${ }^{1, *}$ \\ Departments of ${ }^{1}$ Biochemistry and Molecular Biology, ${ }^{2}$ Biomedical Sciences, Asan Medical Center, University of Ulsan College of Medicine, \\ Seoul 05505, Korea
}

\begin{abstract}
We have identified a mechanism to diminish the proliferative capacity of cells during cell expansion using human adiposederived stromal cells (hAD-SCs) as a model of replicative senescence. hAD-SCs of high-passage numbers exhibited a reduced proliferative capacity with accelerated cellular senescence. Levels of key bioactive sphingolipids were significantly increased in these senescent hAD-SCs. Notably, the transcription of sphingosine kinase 1 (SPHK1) was down-regulated in hAD-SCs at high-passage numbers. SPHK1 knockdown as well as inhibition of its enzymatic activity impeded the proliferation of hAD-SCs, with concomitant induction of cellular senescence and accumulation of sphingolipids, as seen in high-passage cells. SPHK1 knockdown-accelerated cellular senescence was attenuated by co-treatment with sphingosine-1-phosphate and an inhibitor of ceramide synthesis, fumonisin $B_{1}$, but not by treatment with either one alone. Together, these results suggest that transcriptional down-regulation of SPHK1 is a critical inducer of altered sphingolipid profiles and enhances replicative senescence during multiple rounds of cell division. [BMB Reports 2019; 52(3): 220-225]
\end{abstract}

\section{INTRODUCTION}

Sphingolipids are essential components of eukaryotic cells and also exist in the form of some metabolites including sphingomyelins, ceramides, and sphingosines. Besides playing structural roles in cell membranes, they function as bioactive signaling molecules to regulate cell proliferation, differentiation,

*Corresponding authors. Sun-Cheol Choi, Tel: +82-2-3010-2206; Fax: +82-2-3010-5307; E-mail: choisc@amc.seoul.kr; Seong Who Kim, Tel: +82-2-3010-4270; Fax: +82-2-477-4266; E-mail: swhokim@amc.seoul.kr

https://doi.org/10.5483/BMBRep.2019.52.3.012

Received 7 January 2019, Revised 16 January 2019, Accepted 28 January 2019

Keywords: Human adipose-derived stromal cells, Replicative senescence, Sphingolipid, Sphingosine kinase 1, SPHK1 transcription migration, and apoptosis $(1,2)$. The sphingolipid metabolic pathway exhibits an intricate network of reactions involving a variety of enzymes. In this pathway, sphingosine is the backbone of all sphingolipids. Sphingomyelins are produced by sphingomyelin synthases using phosphatidylcholine and ceramide (3). Ceramide, a central sphingolipid, is synthesized by a de novo pathway and a salvage pathway through the action of serine palmitoyltransferase and ceramide synthase, respectively (4). Sphingosine kinase (SPHK) 1 and 2, major enzymes in sphingolipid metabolism, catalyze the phosphorylation of sphingosine to generate sphingosine-1-phosphate (S1P). S1P acts as a bioactive molecule to bind to five specific G-protein-coupled receptors (S1P receptors), thus regulating various intracellular signaling pathways during cell proliferation and migration (5-8).

Several pieces of evidence have demonstrated that sphingolipids play crucial roles in the regulation of the lifespan in model organisms, such as worms, flies, and yeast (8). These lipid species have also emerged as critical regulators of replicative senescence at the cellular level. Replicative cellular senescence causes the proliferative capacity of cells to progressively diminish during their repeated replication $(9$, 10). At this senescent stage, cells lose their replicative potential, leading to gradual impairment of their physiological functions (11). In addition, senescent cells are resistant to diverse stimuli, such as mitogenic and apoptotic stimuli, though they are still viable and metabolically active (12). Generally, senescence triggers morphological changes, such as enlarged and flattened cellular shapes and induces a tumor suppressor network involving the ARF/p53 and p16/pRb pathways, which are used as biomarkers to identify senescent cells (13-15). ARF promotes the stabilization of p53 protein followed by increased expression of $\mathrm{p} 21$, thereby contributing to activation of the $\mathrm{p} 16 / \mathrm{pRb}$ pathway. Thus, the interaction between these two tumor suppressor pathways maintains the senescent state of cells (16-18).

Human adipose-derived stromal cells (hAD-SCs) have been proposed as an attractive cell type for cell-based therapies and regenerative medicine. However, hAD-SCs have a drawback related to their in vitro cell expansion, which is the difficulty of generating sufficient numbers of cells for repeated clinical applications, possibly arising from the replicative senescence

ISSN: 1976-670X (electronic edition)

Copyright (C) 2019 by the The Korean Society for Biochemistry and Molecular Biology

c) This is an open-access article distributed under the terms of the Creative Commons Attribution Non-Commercial License (http://creativecommons.org/licenses/by-nc/4.0) which permits unrestricted non-commercial use, distribution, and reproduction in any medium, provided the original work is properly cited. 
that occurs during continuous cell expansion. To circumvent this cell expansion limitation, we sought to elucidate the molecular mechanisms underlying the diminished proliferative capacity of hAD-SCs. Recent studies have implicated ceramides or sphingosines in cellular senescence (8). SPHKs have also been shown to contribute to cell survival, proliferation, and resistance to apoptosis $(19,20)$, though their roles in cellular senescence remain to be investigated. In this study, we have found that alteration of sphingolipid profiles occurs during the senescence of hAD-SCs. Notably, both changes in the levels of sphingolipids and cellular senescence are caused by transcriptional down-regulation of SPHK1, which is entailed by repeated cell-cycle progression.

\section{RESULTS}

\section{Continuous expansion of hAD-SCs induces cellular} senescence and accumulation of sphingolipids

To identify the precise molecular mechanisms by which the proliferative capacity of hAD-SCs is reduced during in vitro cell expansion, we checked the proliferation rates of these cells undergoing repeated cell division. BrdU incorporation was used to assay DNA synthesis/cell cycle progression in early ( 3 or 4 passages)- and late (17 or 18 passages)-passage cells. As expected, hAD-SCs of high-passage numbers had markedly diminished proliferation rates as demonstrated by incorporation of less BrdU into late-passage cells than early-passage cells (Fig. 1A). The late-passage cells exhibited a higher activity of senescence-associated- $\beta$-galactosidase (SA- $\beta$-gal) than early-passage cells (Fig. 1B, C). Consistently, the levels of cellular senescence markers, including phospho-p53 and p21, were highly enhanced in the late-passage cells, compared to the early-passage cells (Fig. 1D). In contrast, the level of phosphorylated $\mathrm{pRb}$, which allows cell cycle progression, was lower in the late-passage cells than in the early-passage cells (Fig. 1D). Together, these results indicate that hAD-SCs undergo cellular senescence during continuous in vitro expansion.

Accumulating evidence has revealed the critical roles of bioactive sphingolipids in the mechanism of mammalian cell senescence (8). Thus, we hypothesized that these lipid metabolites could function as potential mediators of hAD-SCs senescence. To test this assumption, we first used liquid chromatography-tandem mass spectrometry (LC-MS/MS) to identify the most abundant subspecies of ceramide and sphingomyelin in hAD-SCs. As shown in Supplementary Fig. S1, C16 ceramide and 16:0 sphingomyelin were found to be the major subspecies in these cells. We next examined whether changes in the cellular levels of sphingolipids, including these main subspecies, would occur in hAD-SCs, depending on their passage numbers. Importantly, the levels of key sphingolipid species, C16 ceramide, 16:0 sphingomyelin, sphinganine, and sphingosine, were significantly higher in the late-passage cells than those in the early-passage cells (Fig. 1E).

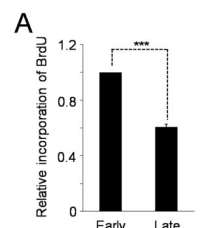

D

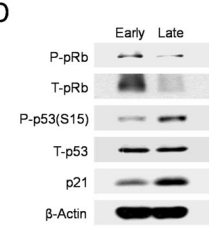

$\mathrm{B}$

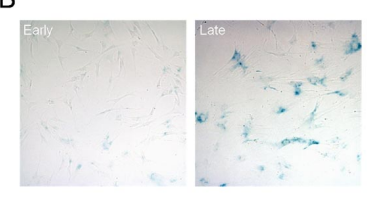

C

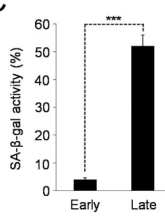

$\mathrm{E}$

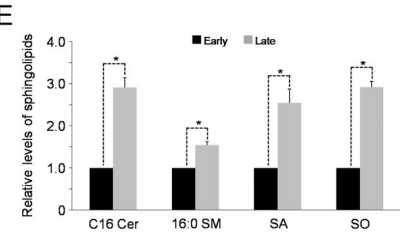

Fig. 1. High passage hAD-SCs undergo cellular senescence and changes in sphingolipid profile. (A) BrdU incorporation assay showing the reduced proliferative capacity of late-passage cells relative to early-passage cells. Error bars indicate the standard error (SE). Asterisks above the bars denote $* * * \mathrm{P}<0.001$ compared to early-passage cells. (B) Late-passage hAD-SCs display higher activity of senescence-associated- $\beta$-galactosidase (SA- $\beta$-gal) than early-passage cells. (C) Quantification of activities of SA- $\beta$-gal in early- and late-passage hAD-SCs. For this, the number of SA- $\beta$-gal positive cells per field was counted. $* * * P<0.001$. (D) Cellular levels of senescence markers, phospho-p53 and p21 are increased in late-passage cells, compared to those in early-passage cells, as analyzed by Western blotting. $\beta$-Actin serves as a loading control. (E) Elevation of levels of sphingolipid species in senescent late-passage hAD-SCs. Cer, ceramide; SM, sphingomyelin; SA, sphinganine; SO, sphingosine. ${ }^{*} \mathrm{P}<0.05$.

Taken together, these results suggest that multiple rounds of cell division induce the accumulation of anti-growth bioactive sphingolipids, such as ceramide and sphingosine, resulting in replicative senescence in the hAD-SCs.

\section{Reduced transcription of SPHK1 in senescent hAD-SCs}

The alteration of sphingolipid profiles, as shown above, indicates the possibility that the expression and/or activity of the sphingolipid metabolic enzymes could be changed in senescent hAD-SCs. Therefore, we compared the expression levels of several sphingolipid metabolic enzymes in the late-passage cells with those in the early-passage cells. Of note, quantitative RT-PCR analysis revealed that higherpassage hAD-SCs had significantly reduced levels of sphingosin kinase 1 (SPHK1) mRNA, compared to those in lower-passage cells (Fig. 2B). In contrast, neither SPHK2, an isoform of SPHK1, nor the other key sphingolipid metabolic genes, exhibited marked changes in the amount of each transcript as the passage number increased. Consistent with the lowered level of mRNA, the expression of SPHK1 protein was also highly decreased in late-passage cells, compared to early-passage cells (Fig. 2C, D). Therefore, these results suggest that transcription of SPHK1 is down-regulated during hAD-SCs senescence. 
A

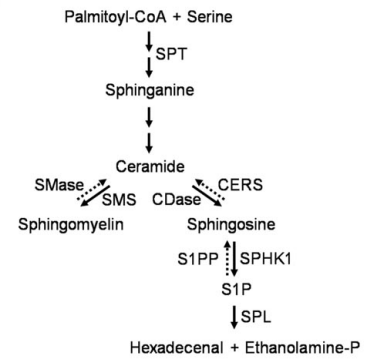

B

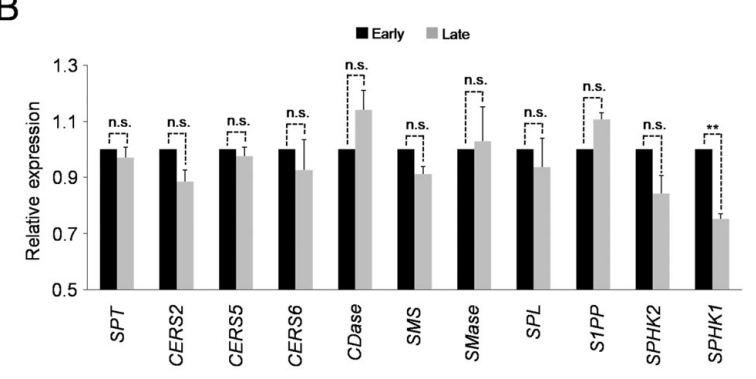

Fig. 2. Transcription of SPHK1 is reduced in senescent hAD-SCs. (A) Scheme of sphingolipid metabolism. SPT, serine palmitoyltransferase; SMase, sphingomyelinase; SMS, sphingomyelin synthase; CDase, ceramidase; CERS, ceramide synthase; SPHK1, sphingosine kinase 1; S1PP, sphingosine-1-phosphate phosphatase; SPL, sphingosine-1-phosphate lyase. (B) Late-passage hAD-SCs have lower levels of SPHK1 mRNA than early-passage cells as shown by qRT-PCR. ${ }^{* * P}<0.01$ compared to early-passage cells. n.s. not significant. (C) Relative expression of SPHK1 protein in earlyand late-passage cells. $\beta$-Actin is a loading control. (D) Quantification of SPHK1 protein levels in (C) (normalized to $\beta$-Actin). *P $<0.05$.

\section{Silencing of SPHK1 leads to cellular senescence and accumulation of sphingolipids}

Given that SPHK1 is a key regulator of the balance of anti-growth sphingolipids, ceramide and sphingosine, and pro-growth sphingolipid, S1P (21), it is possible that transcriptional down-regulation of SPHK1 might be a critical inducer of the accumulation of sphingolipids and cellular senescence in high-passage hAD-SCs. To test this possibility, we carried out lentiviral shRNA-mediated silencing of SPHK1, or repressed its enzymatic activity, to examine the sphingolipid profile and cellular senescence in cells lacking the action of SPHK1. We tested the efficacies of three shRNAs specific for SPHK1 (designated shSPHK1 \#1, \#2 or \#3) by Western blot analysis. Although all three shRNAs silenced SPHK1 (Fig. 3A), we used shSPHK1 \#2 for further experiments because it was more effective compared to the other two shRNAs. Knockdown of SPHK1 as well as treatment with SKI, a SPHK1 inhibitor, significantly reduced the incorporation of BrdU into cellular DNA compared to control cells (Fig. 3B; Supple-
A

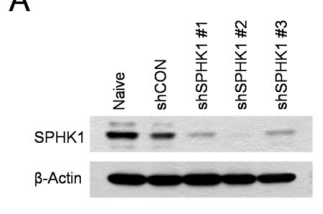

C

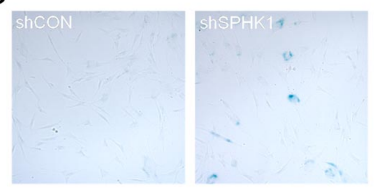

E

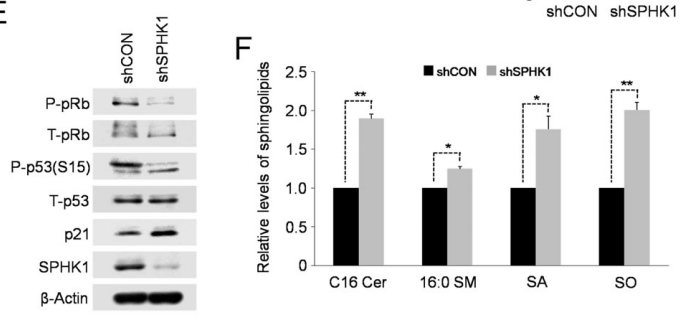

$\mathrm{B}$

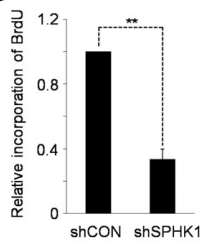

D

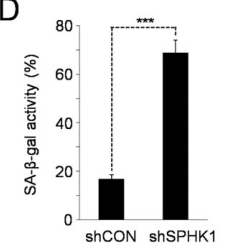

Fig. 3. Knockdown of SPHK1 promotes cellular senescence and accumulation of sphingolipids. (A) Western blots showing knockdown efficacies of shRNAs against SPHK1 (shSPHK1 \#1-3). shCON, a negative control shRNA. (B) Proliferative capacity is decreased in SPHK1-depleted hAD-SCs. $* * P<0.01$ compared to control shRNA-expressing cells. (C) Cellular senescence is enhanced in SPHK1-silenced hAD-SCs as shown by relatively higher activity of SA- $\beta$-gal. (D) Quantification of activities of SA- $\beta$-gal in shCON or shSPHK1-expressing hAD-SCs. $* * * \mathrm{P}<$ 0.001 . (E) Depletion of SPHK1 causes an increase in the levels of senescence markers. $\beta$-Actin serves as a loading control. (F) Elevated levels of key sphingolipid species induced by knockdown of SPHK1. ${ }^{* P}<0.05$ and $* * P<0.01$, compared to control shRNA-expressing cells.

mentary Fig. S2A), indicative of the lowered proliferative ability. As with high-passage cells, SPHK1-depleted or SKI-treated hAD-SCs underwent accelerated cellular senescence as evidenced by higher SA-b-gal activity (Fig. 3C, D; Supplementary Fig. S2B, C). In support of this, the levels of cellular senescence markers, phospho-p53 and p21, were up-regulated in the SPHK1-silenced or SKI-treated cells and the level of phospho-pRb, a marker of cell proliferation, was reduced (Fig. 3E; Supplementary Fig. S2D). Furthermore, LC-MS/MS analysis revealed elevated levels of key sphingolipids, including ceramide and sphingosine, in SPHK1-depleted cells compared to negative control shRNA-expressing cells (Fig. 3F). siRNA-mediated silencing of SPHK1 has been shown to induce apoptosis in MCF-7 breast cancer cells (22). Thus, we also checked whether treatment with SKI could induce this programmed cell death in hAD-SCs. In contrast, annexin $\mathrm{V}$ staining revealed no significant increase in the number of apoptotic cells in SKI-treated hAD-SCs 
(Supplementary Fig. S2E). Thus, it seems likely that down-regulation of SPHK1 expression or activity induces cellular senescence and/or apoptosis depending on the cell types and cell context. Overall, these data support the conclusion that reduced transcription of SPHK1 is responsible for altered sphingolipid profiles and cellular senescence elicited by continuous cell expansion.

\section{Alteration of sphingolipid profiles causes cellular senescence in SPHK1-depleted cells}

While ceramide and sphingosine are usually implicated in the induction of cell-cycle arrest, apoptosis, and senescence, sphingosine-1-phosphate (S1P) promotes cell proliferation, survival, and migration (23). Thus, we speculated that cellular senescence accelerated by SPHK1 knockdown might be due to increased ceramide levels and/or decreased S1P levels. To test this hypothesis, we investigated whether SPHK1 knockdown-induced cellular senescence could be attenuated by inhibition of ceramide synthesis or exogenous supplementation with S1P during cell culture. BrdU incorporation assays showed that the proliferation impeded by shRNAmediated silencing of SPHK1 could not be recovered by single treatment with $\mathrm{S} 1 \mathrm{P}$ or fumonisin $\mathrm{B}_{1}\left(\mathrm{FB}_{1}\right)$, a ceramide synthesis inhibitor, and even by co-treatment with both (Fig. 4A; Supplementary Fig. S3A, S4A), indicating the irreversibility of
A

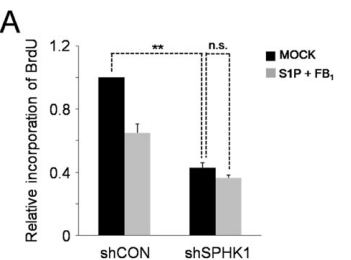

B

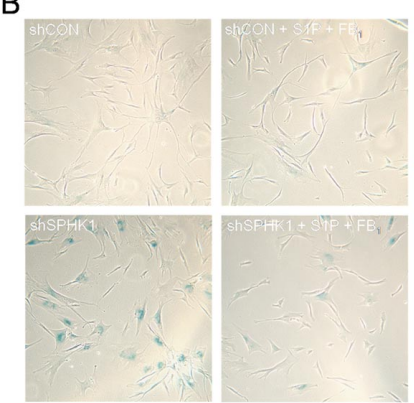

Fig. 4. SPHK1 knockdown-accelerated cellular senescence is attenuated by inhibition of ceramide synthesis and concurrent supplementation with S1P. (A) No rescue of reduced proliferation capacity of SPHK1-depleted hAD-SCs by co-treatment with S1P and $\mathrm{FB}_{1}$. $* * \mathrm{P}<0.01$. (B) Co-treatment with $\mathrm{S} 1 \mathrm{P}$ and $\mathrm{FB}_{1}$ lowers the activity of SA- $\beta$-gal enhanced by depletion of SPHK1. (C) Quantification of activities of SA- $\beta$-gal in shCON or shSPHK1expressing hAD-SCs which were co-treated with $\mathrm{S} 1 \mathrm{P}$ and $\mathrm{FB}_{1}$ or untreated. $* * * \mathrm{P}<0.001$ cellular senescence. In contrast, the elevated activity of SA- $\beta$-gal in SPHK1-silenced cells was reduced by simultaneous co-treatment with $\mathrm{S} 1 \mathrm{P}$ and $\mathrm{FB}_{1}$, but not by single treatment with either one alone (Fig. 4B, C; Supplementary Fig. S3B, C and $\mathrm{S} 4 \mathrm{~B}, \mathrm{C})$, suggesting that cellular senescence accelerated by SPHK1 depletion may result from increase in ceramide levels with concurrent decrease in S1P levels. Taking together, we conclude that depletion of SPHK1 alters the balance of pro-death and anti-death bioactive sphingolipids, enhancing replicative cellular senescence.

\section{DISCUSSION}

Recent studies have demonstrated that sphingolipids, such as ceramide and sphingosine, and their metabolic enzymes are involved in the regulation of cellular senescence in various cell types (8). However, how changes in the levels of sphingolipid species and activities of their metabolic enzymes are induced in cells undergoing replicative senescence remains elusive. In this study, we showed that downregulation of SPHK1 expression at the transcriptional level acts as a key inducer of altered sphingolipid profiles, accelerating cellular senescence during cell expansion. Like most human cells, human adipose tissue-derived stromal cells, a model of replicative senescence used in this work, exhibited a decrease in proliferative capacity with a concomitant enhancement of cellular senescence as they underwent increasing passages. Previously, human senescent fibroblasts of high-passage numbers were shown to have increased ceramide levels and sphingomyelinase (SMase) activity, compared to those of lower passage numbers (5). Of note, senescent hAD-SCs of high-passage numbers have significantly elevated levels, not only of ceramide, but of other sphingolipids, including sphingomyelin, sphingosine, and sphinganine (Fig. 1). In addition, among several sphingolipid metabolic enzymes, only the expression of SPHK1 was significantly down-regulated at the mRNA level as well as at the protein level in senescent hAD-SCs. In support of a pivotal role for this decreased expression of SPHK1 in inducing senescence, shRNA-mediated silencing of SPHK1 promoted cellular senescence at the expense of proliferation and up-regulated the levels of the same sphingolipid species as observed in late-passage cells. Pharmacological inhibition of SPHK1 activity suppressed cell growth, but accelerated cellular senescence. Furthermore, the enhanced cellular senescence in SPHK1-depleted cells could be attenuated by exogenous addition of S1P with concurrent inhibition of ceramide synthesis. Given these findings, it is possible that reduced transcription of SPHK1 may function as a bona fide inducer of replicative senescence through regulation of sphingolipid metabolism during repeated rounds of cell division.

Cellular levels of SPHK1 expression have been shown to play key roles in the sphingolipid-mediated determination of cell fates. The levels of SPHK1 mRNA are generally increased 
in various types of human cancers (24). In line with this, overexpression of SPHK1 promotes focus formation, growth of cells in soft agar, and tumor formation in NOD/SCID mice (25), pointing to its oncogenic role. However, it also reduces cellular apoptosis (26). Increased levels of S1P by overexpression of SPHK1 expedite cell-cycle progression and enhance cell growth, whereas overexpression of S1P phosphatase increases cell death (27). Thus, it appears that the up-regulation of SPHK1 expression leads to pro-growth and anti-apoptotic signals mediated by the SPHK1/S1P pathway. Down-regulation of SPHK1 expression provides a mechanism of tumor suppression by modulating the levels of sphingolipid species as shown in studies of double-KO mice lacking the tumor suppressor p53 gene and SPHK1. p53-null mice developed thymic lymphomas, which were completely abrogated by deletion of SPHK1 in these mice (28). In p53-KO mice, expression of SPHK1 and levels of S1P were elevated, whereas levels of ceramide were decreased, compared to wild-type mice. These sphingolipid profiles in p53-null mice were reversed by deletion of SPHK1, in turn accompanied by cellular senescence but not apoptosis. Notably, SPHK1 has been shown to undergo proteolytic degradation in response to genotoxic stress in a p53-dependent manner (29). These results suggest that p53 induces its tumor suppressor effects by down-regulating expression of SPHK1 at the posttranslational level, leading to decreased levels of S1P and increased levels of ceramide and sphingosine, which then mediate cellular senescence. As shown by our results, down-regulation of SPHK1 expression at the transcriptional level caused similarly elevation of ceramide and sphingosine levels, contributing to replicative cellular senescence. Given these results, it is tempting to speculate that the mode of regulation of SPHK1 expression to induce cellular senescence depends on upstream inducers and the physiological context. It seems likely that for tissue homeostasis, a rapid response mechanism for inducing senescence, such as p53-mediated proteolysis of SPHK1, is necessary for cells to prevent immediately tumorigenesis in response to genotoxic stresses, including DNA damageinducing agents and oncogenic activation. In contrast, a relatively slower mechanism, such as down-regulation of SPHK1 transcription, may be appropriate for inducing replicative senescence in response to telomere shortening and oxidative stress caused by multiple rounds of cell division. However, it cannot be excluded that both the rapid and slow mechanisms might function in parallel to prevent tumor formation and/or induce replicative senescence. More than one mode of regulation would ensure a robust mechanism to induce cellular senescence in the right place at the right time during tissue homeostasis and aging.

As sphingolipid species, including sphingomyelin, ceramide, sphingosine, and S1P, are interconvertible, knockdown of one sphingolipid metabolic enzyme would alter the relative levels of these metabolites. Furthermore, the levels of activity of a sphingolipid metabolic enzyme could affect those of other enzymes. Overexpression of S1P phosphatase-1, which dephosphorylates S1P, enhances de novo synthesis of ceramide, possibly owing to de-repression of serine palmitoyItransferase or ceramide synthase by S1P (21). Treatment with fumonisin $B_{1}$, an inhibitor of ceramide synthase, has been shown to increase the activity of acidic sphingomyelinase, accompanied with a decrease of sphingomyelin, and the expression and activities of serine palmitoyltransferase and SPHK1 in mice livers (30). Thus, a 'sphingolipid rheostat' has been proposed to regulate the relative levels of sphingolipid metabolites, thereby determining cell fates (21). SPHK1 acts as a critical regulator of the sphingolipid rheostat to maintain a balance between pro-growth and pro-death bioactive sphingolipids. As shown by our assays, knockdown of SPHK1 seemed to tilt the balance of ceramide, sphingosine, and S1P in favor of growth inhibition and cell death. SPHK1 knockdown-reduced proliferative capacity could be recovered by neither co-treatment with both S1P and a ceramide synthesis inhibitor, fumonisin $B_{1}$, nor treatment with either one, indicative of the irreversibility of cellular senescence accelerated by depletion of SPHK1. However, this cellular senescence could be attenuated efficiently by co-treatment with both the reagents, but not by treatment with either one alone, suggesting the crucial roles that increased ceramide levels and decreased S1P levels each play in promoting replicative senescence in SPHK1-depleted cells. In most cell types, the cellular levels of ceramide, sphingosine, and S1P differ approximately by an order of magnitude, with ceramide being present at the highest and S1P at the lowest level (2). A small change in ceramide can lead to profound changes in S1P. In addition, the levels of ceramide in SPHK1-depleted cells are about two times higher than those in normal control cells (Fig. 3). Despite these facts, inhibition of ceramide generation alone was unable to attenuate acceleration of cellular senescence by knockdown of SPHK1, thus revealing the strong potency of low levels of S1P in shifting the balance between pro- and anti-death signals toward cell survival.

In conclusion, this study found that transcriptional down-regulation of SPHK1 serves as a critical inducer of a tilt in the balance between pro- and anti-growth bioactive sphingolipids in favor of growth inhibition and cellular senescence during in vitro cell expansion. Further studies are warranted to elucidate the molecular mechanisms by which SPHK1 expression is negatively regulated at the transcriptional level during multiple rounds of cell divisions. Since SPHK1 is an essential regulator of the sphingolipid rheostat, better understanding of the control of its expression holds promise for the development of novel tools to prolong the proliferative capacity of cells for cell-based therapy and regenerative medicine.

\section{MATERIALS AND METHODS}

Detailed information can be found in the Supplementary data. 


\section{ACKNOWLEDGEMENTS}

This work was supported by the National Research Foundation of Korea (NRF) MRC grant funded by the Korean government (MSIT) (2018R1A5A2020732) and by Basic Science Research Program through the National Research Foundation of Korea funded by the Ministry of Science, ICT \& Future Planning (2016R1A2B4013355).

\section{CONFLICTS OF INTEREST}

The authors have no conflicting interests.

\section{REFERENCES}

1. Goni FM and Alonso A (2006) Biophysics of sphingolipids I. Membrane properties of sphingosine, ceramides and other simple sphingolipids. Biochim Biophys Acta 1758, 1902-1921

2. Hannun YA and Obeid LM (2008) Principles of bioactive lipid signalling: lessons from sphingolipids. Nat Rev Mol Cell Biol 9, 139-150

3. Adada M, Luberto C and Canals D (2016) Inhibitors of the sphingomyelin cycle: Sphingomyelin synthases and sphingomyelinases. Chem Phys Lipids 197, 45-59

4. Hernandez-Corbacho MJ, Salama MF, Canals D, Senkal CE and Obeid LM (2017) Sphingolipids in mitochondria. Biochim Biophys Acta Mol Cell Biol Lipids 1862, 56-68

5. Venable ME, Lee JY, Smyth MJ, Bielawska A and Obeid LM (1995) Role of ceramide in cellular senescence. J Biol Chem 270, 30701-30708

6. Kolesnick RN, Goni FM and Alonso A (2000) Compartmentalization of ceramide signaling: physical foundations and biological effects. J Cell Physiol 184, 285-300

7. Zhang H, Desai NN, Olivera A, Seki T, Brooker G and Spiegel S (1991) Sphingosine-1-phosphate, a novel lipid, involved in cellular proliferation. J Cell Biol 114, 155-167

8. Trayssac M, Hannun YA and Obeid LM (2018) Role of sphingolipids in senescence: implication in aging and age-related diseases. J Clin Invest 128, 2702-2712

9. Campisi J (1997) The biology of replicative senescence. Eur J Cancer 33, 703-709

10. Hayflick L and Moorhead PS (1961) The serial cultivation of human diploid cell strains. Exp Cell Res 25, 585-621

11. Flatt T (2012) A new definition of aging? Front Genet 3, 148

12. Sherr CJ and DePinho RA (2000) Cellular senescence: mitotic clock or culture shock? Cell 102, 407-410

13. Michaloglou C, Vredeveld LC, Soengas MS et al (2005) BRAFE600-associated senescence-like cell cycle arrest of human naevi. Nature 436, 720-724

14. Chen QM, Prowse KR, Tu VC, Purdom S and Linskens $\mathrm{MH}$ (2001) Uncoupling the senescent phenotype from telomere shortening in hydrogen peroxide-treated fibroblasts. Exp Cell Res 265, 294-303

15. Lowe SW, Cepero E and Evan G (2004) Intrinsic tumour suppression. Nature 432, 307-315

16. Serrano $M$, Lin AW, McCurrach ME, Beach D and Lowe SW (1997) Oncogenic ras provokes premature cell senescence associated with accumulation of p53 and p16INK4a. Cell 88, 593-602

17. Stein GH, Beeson M and Gordon L (1990) Failure to phosphorylate the retinoblastoma gene product in senescent human fibroblasts. Science 249, 666-669

18. Wei W, Hemmer RM and Sedivy JM (2001) Role of p14(ARF) in replicative and induced senescence of human fibroblasts. Mol Cell Biol 21, 6748-6757

19. Vadas M, Xia P, McCaughan G and Gamble J (2008) The role of sphingosine kinase 1 in cancer: oncogene or non-oncogene addiction? Biochim Biophys Acta 1781, 442-447

20. Meng H, Yuan $Y$ and Lee VM (2011) Loss of sphingosine kinase 1/S1P signaling impairs cell growth and survival of neurons and progenitor cells in the developing sensory ganglia. PLoS One 6, e27150

21. Spiegel S and Milstien S (2003) Sphingosine-1-phosphate: an enigmatic signalling lipid. Nat Rev Mol Cell Biol 4, 397-407

22. Taha TA, Kitatani K, El-Alwani M, Bielawski J, Hannun YA and Obeid LM (2006) Loss of sphingosine kinase-1 activates the intrinsic pathway of programmed cell death: modulation of sphingolipid levels and the induction of apoptosis. FASEB J 20, 482-484

23. Bartke $\mathrm{N}$ and Hannun YA (2009) Bioactive sphingolipids: metabolism and function. J Lipid Res 50 Suppl, S91-96

24. Johnson KR, Johnson KY, Crellin HG et al (2005) Immunohistochemical distribution of sphingosine kinase 1 in normal and tumor lung tissue. J Histochem Cytochem 53, 1159-1166

25. Xia P, Gamble JR, Wang L et al (2000) An oncogenic role of sphingosine kinase. Curr Biol 10, 1527-1530

26. Olivera A, Kohama T, Edsall L et al (1999) Sphingosine kinase expression increases intracellular sphingosine-1phosphate and promotes cell growth and survival. J Cell Biol 147, 545-558

27. Le Stunff H, Galve-Roperh I, Peterson C, Milstien S and Spiegel S (2002) Sphingosine-1-phosphate phosphohydrolase in regulation of sphingolipid metabolism and apoptosis. J Cell Biol 158, 1039-1049

28. Heffernan-Stroud LA, Helke KL, Jenkins RW, De Costa AM, Hannun YA and Obeid LM (2012) Defining a role for sphingosine kinase 1 in p53-dependent tumors. Oncogene 31, 1166-1175

29. Taha TA, Osta W, Kozhaya L et al (2004) Downregulation of sphingosine kinase- 1 by DNA damage: dependence on proteases and p53. J Biol Chem 279, 20546-20554

30. He Q, Suzuki H, Sharma N and Sharma RP (2006) Ceramide synthase inhibition by fumonisin B1 treatment activates sphingolipid-metabolizing systems in mouse liver. Toxicol Sci 94, 388-397 